\title{
Trapping Surface Electrons on Graphene Layers and Islands
}

\author{
D. Niesner, ${ }^{1}$ Th. Fauster, ${ }^{1}$ J. I. Dadap ${ }^{2}$ N. Zaki, ${ }^{2}$ K. R. Knox ${ }^{2}$ P.-C. \\ Yeh, ${ }^{2}$ R. Bhandari, ${ }^{2}$ R. M. Osgood,${ }^{2}$ M. Petrović, ${ }^{3}$ and M. Kralj ${ }^{3}$ \\ ${ }^{1}$ Lehrstuhl für Festkörperphysik, Universität \\ Erlangen-Nürnberg, D-91058 Erlangen, Germany \\ ${ }^{2}$ Columbia University, New York, New York 10027, USA \\ ${ }^{3}$ Institut za fiziku, Bijenička 46, HR-10000 Zagreb, Croatia
}

\begin{abstract}
We report the use of time- and angle-resolved two-photon photoemission to map the bound, unoccupied electronic structure of the weakly coupled graphene/ $\operatorname{Ir}(111)$ system. The energy, dispersion, and lifetime of the lowest three image-potential states are measured. In addition, the weak interaction between Ir and graphene permits observation of resonant transitions from an unquenched Shockley-type surface state of the Ir substrate to graphene/Ir image-potential states. The image-potential-state lifetimes are comparable to those of mid-gap clean metal surfaces. Evidence of localization of the excited electrons on single-atom-layer graphene islands is provided by coverage-dependent measurements.

PACS numbers: 73.22.Pr, 79.60.Dp, 73.20.-r, 79.20.Ws
\end{abstract}


Graphene on metal surfaces are a materials system of enormous fundamental and applied interest. The graphene/metal interface is encountered in the rapidly expanding technological system of CVD graphene on $\mathrm{Cu}$ foil, in the structurally precise monolayer epitaxial systems of graphene on single-crystal $\mathrm{Ru}, \mathrm{Ir}$, or $\mathrm{Ni}$, and finally in the metal contacts of graphene field-effect transistors or other devices. Questions then arise on the electronic structure of graphene on metal surfaces and in fact several recent studies have addressed questions such as the role of lattice mismatch on band structures. Most studies of the electronic structure of graphene have focused on the band structure in the vicinity of its K point, near the Fermi edge. Further there has been a paucity of measurements about its unoccupied electronic structure and the dynamics of strongly excited electrons. Image-potential states offer one important approach to probe the excited state manifold and are known to vary with interfacial quality, dielectric properties, and electronic structure. In graphene the large band gap at the $\Gamma$ point results in Bragg reflection from the crystal within a certain range of energy and momentum. In fact a recent theoretical study has shown the existence of a dual Rydberg-like series of even and odd symmetry image-potential states in a single free-standing sheet of graphene [1]. Image-potential states on graphene may experience different dynamic constraints. For example, the different phase space for decay in two dimensions compared to three dimensions may affect the lifetimes for electrons trapped in image-potential states on graphene. In fact, more generally the response of the image electron to the composite dielectric/metal systems is itself of basic physics interest.

In this Letter, we investigate the uncharted region of the bound, unoccupied electronic structure of epitaxial graphene grown on $\operatorname{Ir}(111)$ in the vicinity of the graphene $\Gamma$ point; our measurements are made via the image-potential states using angle- and time-resolved two-photon photoemission (2PPE) as indicated by arrows in Fig. 1. This system was chosen for several reasons: First, because of the weak coupling in the graphene/Ir(111) system, the electronic structure of the graphene overlayer is nearly intact, with sharp Dirac dispersion characteristics [2]. In addition, the moiré corrugation of the epitaxial graphene on $\operatorname{Ir}(111)$ has been found to be only $0.35 \pm 0.10 \AA$ based on atomic force microscopy measurements [3] indicating a smooth epitaxial graphene surface. Second, the molecular-based growth is well characterized and saturates at precisely one monolayer (ML) of epitaxial graphene [4]. Our results show that image-potential states may be excited from the Ir/graphene interfacial region and have binding energies and lifetimes comparable to those of mid-gap clean metal 


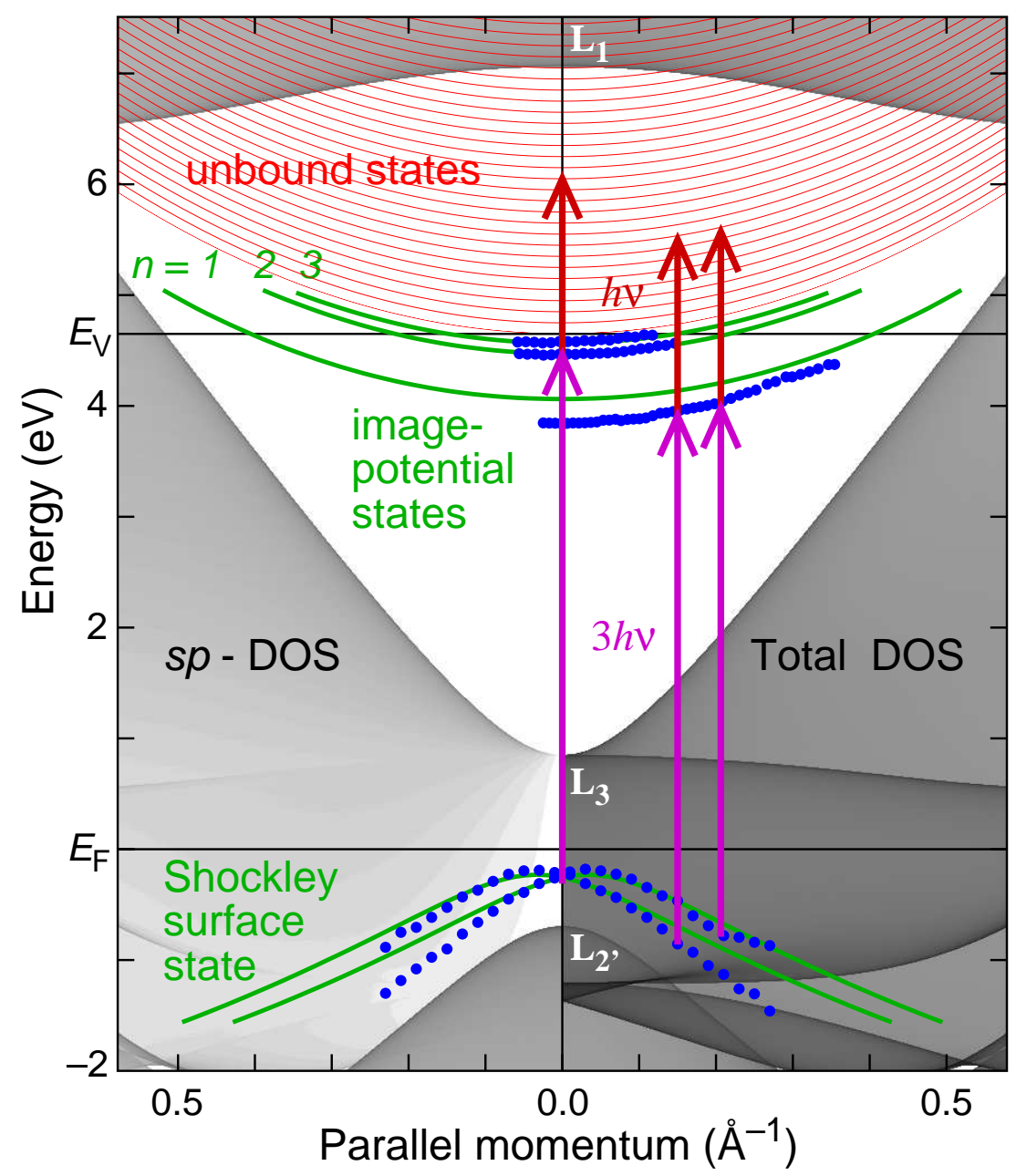

FIG. 1. (Color online). Arrows indicate 2PPE transitions between surface and image-potential states. The experimental results (dots) are compared to calculations (lines). The projected bulkband structure of $\operatorname{Ir}(111)$ along the $\Gamma K$ direction is shaded according to the total and sp-density of states (DOS) at the right and left, respectively.

surfaces. In addition, spectral measurements of binding energy versus coverage show clearly that at low graphene coverage, image-potential electrons are trapped on graphene islands by surface work function differences between the metal and graphene regions, an observation of high importance for understanding of transport at graphene-metal interfaces [5].

Our choice of two-photon photoemission is the result of its high temporal and energy resolution. Other experimental observations of image-potential states have used scanning tunneling spectroscopy, i. e., graphene on $\mathrm{SiC}[6]$ and on $\mathrm{Ru}(0001)$ [7]. This technique, however, measures the image-potential series in the presence of strongly distorting electric 
field between tip and sample and without time-resolved possibilities.

The experiments were conducted using monochromatic and bichromatic 2PPE, and angleresolved photoemission (ARPES). Details of the monochromatic 2PPE setup at Columbia which was used in the photon energy range of $3.8<h \nu<4.9 \mathrm{eV}$ are given in Ref. [8]. Bichromatic and time-resolved 2PPE measurements were performed in Erlangen using pump-probe methods with the third harmonic (UV) and the fundamental (IR, $1.51<h \nu<1.62 \mathrm{eV})$ as described in Ref. [9]. Additionally, occupied-state ARPES measurements were performed at APE (ELETTRA) using a photon energy of $55 \mathrm{eV}$ with an energy resolution of $20 \mathrm{meV}$. The resolution of the $2 \mathrm{PPE}$ experiments was $40 \mathrm{meV}$. The base pressure in all three ultrahigh vacuum (UHV) systems was better than $1 \times 10^{-8} \mathrm{~Pa}$. All measurements used $p$-polarized beams.

Graphene was prepared by cycles of temperature programmed growth, TPG (room temperature ethene exposure $6 \times 10^{-6} \mathrm{~Pa}$ for $60 \mathrm{~s}$ and flashed to $\approx 1450 \mathrm{~K}$ ), followed by a chemical vapor deposition run $\left(6 \times 10^{-6} \mathrm{~Pa}\right.$ of ethene for $300 \mathrm{~s}$ at $\left.1150 \mathrm{~K}\right)$, to form exactly one graphene monolayer [4]. Growth was monitored by low-energy electron diffraction (LEED) after each cycle, which showed the development of the characteristic moiré pattern of uniformly oriented graphene [10], as graphene coverage varied from 0 to 1 ML; LEED patterns (not shown) revealed these patterns clearly.

Figure $2 \mathrm{a}$ shows the measured 2PPE intensity obtained at $1 \mathrm{ML}$ and for $h \nu=1.59 \mathrm{eV}$ along the $\Gamma \mathrm{K}$ direction. Three unoccupied bands are observed. The pumping process could be deduced from its photon-energy dependence, thus in bichromatic case, all peak positions shifted linearly with IR photon energy, indicating that the process involves pumping by a UV photon and photoemission by an IR photon [11]. All 2PPE features vanished when the IR beam was switched to $s$-polarization, indicating the expected symmetry for image-potential states. The effective masses of all three states are $0.9 \pm 0.1 m_{e}$. The binding energies of the three states with respect to the vacuum level are given in Table II. The measured energies and effective masses are close to the free electron mass and fit well to a Rydberg-like-series of image-potential states with a nonvanishing quantum defect [12].

Figure 2a shows that the $n=1$ band is most intense for parallel momenta $k_{\|}$between 0.08 and $0.17 \AA^{-1}$ [cf. points in Fig. 2a], with the intensity typically decreasing monotonically with increasing $k_{\|}[13]$. Direct transitions from initial surface bands can lead to intensity resonances [8]. In order to identify possible initial states for $2 \mathrm{PPE}$, we have performed 


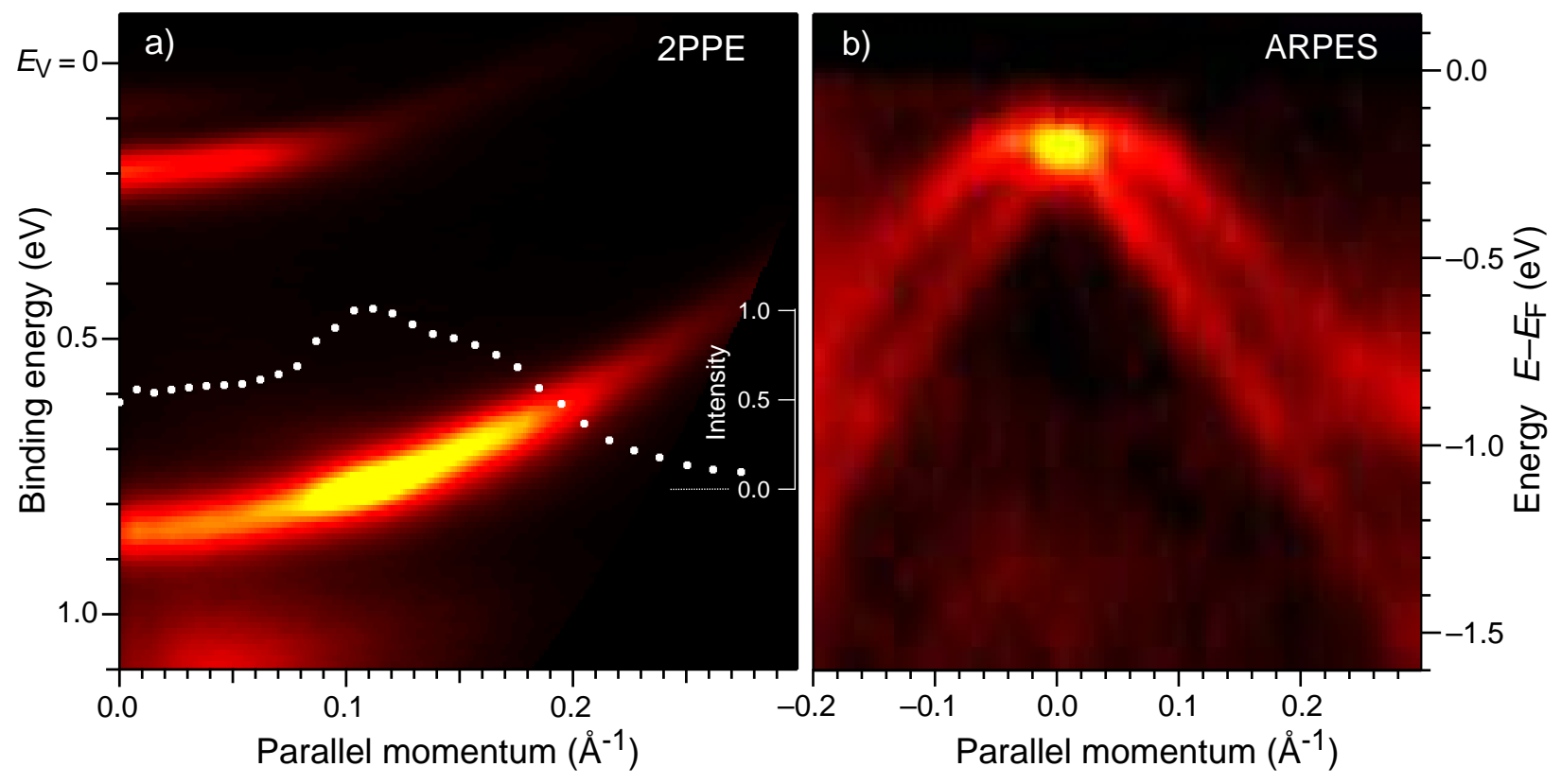

FIG. 2. (Color online). a) Intensity map of the $2 \mathrm{PPE}$ signal recorded with photon energy $h \nu=$ $1.59 \mathrm{eV}$ for $1 \mathrm{ML}$ graphene on $\operatorname{Ir}(111)$. Points represent the intensity of the lowest $n=1$ band. b) ARPES map showing initial states for $h \nu=55 \mathrm{eV}$.

ARPES measurements of graphene on $\operatorname{Ir}(111)$. The ARPES data in Fig. 2b show two parabolic-like dispersions with a downward curvature. The two branches are shifted from $k_{\|}=0$ by $\pm 0.033 \pm 0.001 \AA^{-1}$ and have a maximum energy of $-0.19 \pm 0.01 \mathrm{eV}$. Similar results were also obtained with the fourth harmonic $(6.2 \mathrm{eV})$ in the $2 \mathrm{PPE}$ setup. Rashbatype splittings of similar magnitude are found in other systems, e.g., a Bi/Ag(111) surface alloy [14]. These bands are also observed on clean $\operatorname{Ir}(111)$ [15, 16] indicating that this surface feature is inherent to the clean metal surface. The surface-state energy reported for clean and graphene-covered surfaces differs by about $0.2 \mathrm{eV}$, an effect, which is consistent with a charge transfer between substrate and overlayer and which can shift graphene [16] or iridium states [17, 18].

The initial band dispersion is plotted together with the measured dispersion of the imagepotential states in Fig. 1 (blue dots). The arrows connecting initial states to the $n=1$ image-potential band are at slightly larger $k_{\|}$values than the enhanced intensity in Fig. 2a. In the absence of resonances the 2PPE intensity along image-potential bands decreases continuously with increasing parallel momentum [13]. In the present case due to the finite energy and angle resolution the intensity maxima are shifted to lower $k_{\|}$values compared 
TABLE I. Experimental and calculated binding energies and lifetimes for image-potential states on graphene/ $\operatorname{Ir}(111)$.

\begin{tabular}{lccc}
\hline \hline$n$ & $E_{n}^{\exp }(\mathrm{eV})$ & $E_{n}^{\text {calc }}(\mathrm{eV})$ & $\tau(\mathrm{fs})$ \\
\hline 1 & $0.83 \pm 0.02$ & 0.59 & $35 \pm 3$ \\
2 & $0.19 \pm 0.02$ & 0.18 & $114 \pm 6$ \\
3 & $0.09 \pm 0.02$ & 0.08 & $270 \pm 12$ \\
\hline \hline
\end{tabular}

to the position found in the dispersion analysis. The additional resonance into the $n=2$ band (see Fig. 1) can be inferred from the similar intensity as for the $n=1$ state at $k_{\|}=0$ in Fig. 2a and is confirmed by photon-energy-dependent data presented in the supplemental material [19].

In order to understand the character of the initial state, we calculated the projected bulkband structure of $\operatorname{Ir}(111)$ using a non-relativistic parameterized tight-binding scheme [20]. Figure 1 shows this projected structure along the $\Gamma K$ direction, at the right. The shading represents the one-dimensional density of states (1D-DOS). The left part of Fig. 1 shows the 1D-DOS of bands according to their $s p$-character. For $k_{\|}=0$, the lower edge of the $s p$-band gap is at $-0.7 \mathrm{eV}$, which corresponds to the $L_{2^{\prime}}$ point. The band edge of the total projected bulk-band structure disperses upward from the $L_{3}$ point around $+0.8 \mathrm{eV}$ and picks up $s p$ contributions. On the other hand the lower sp-band edge shows a downward dispersion. The energy of the Shockley surface state was calculated using the $s p$-band edges within a scattering model [21]. The calculations used the experimental work function of $4.65 \mathrm{eV}$ for graphene on $\operatorname{Ir}(111)$. The calculated bands were shifted by $\pm 0.033 \AA^{-1}$ to account for the experimentally observed Rashba splitting and are drawn as green lines in Fig. 1] in the region below the Fermi energy. The experimentally extracted dispersion shown by dots agrees well with the calculation. The Shockley-type surface state is apparently not quenched by the graphene layer at a distance of $3.4 \AA[22]$, because its probability density is concentrated at the $\operatorname{Ir}(111)$ surface.

The scattering model was also used to calculate the energies of the image-potentialband series [21]. The calculated binding energies, given in Table I, are approximately those expected for states located near midgap (see Fig. 11). However, the calculated $n=1$ binding energy is significantly smaller than the experimental value. This discrepancy is due to the 

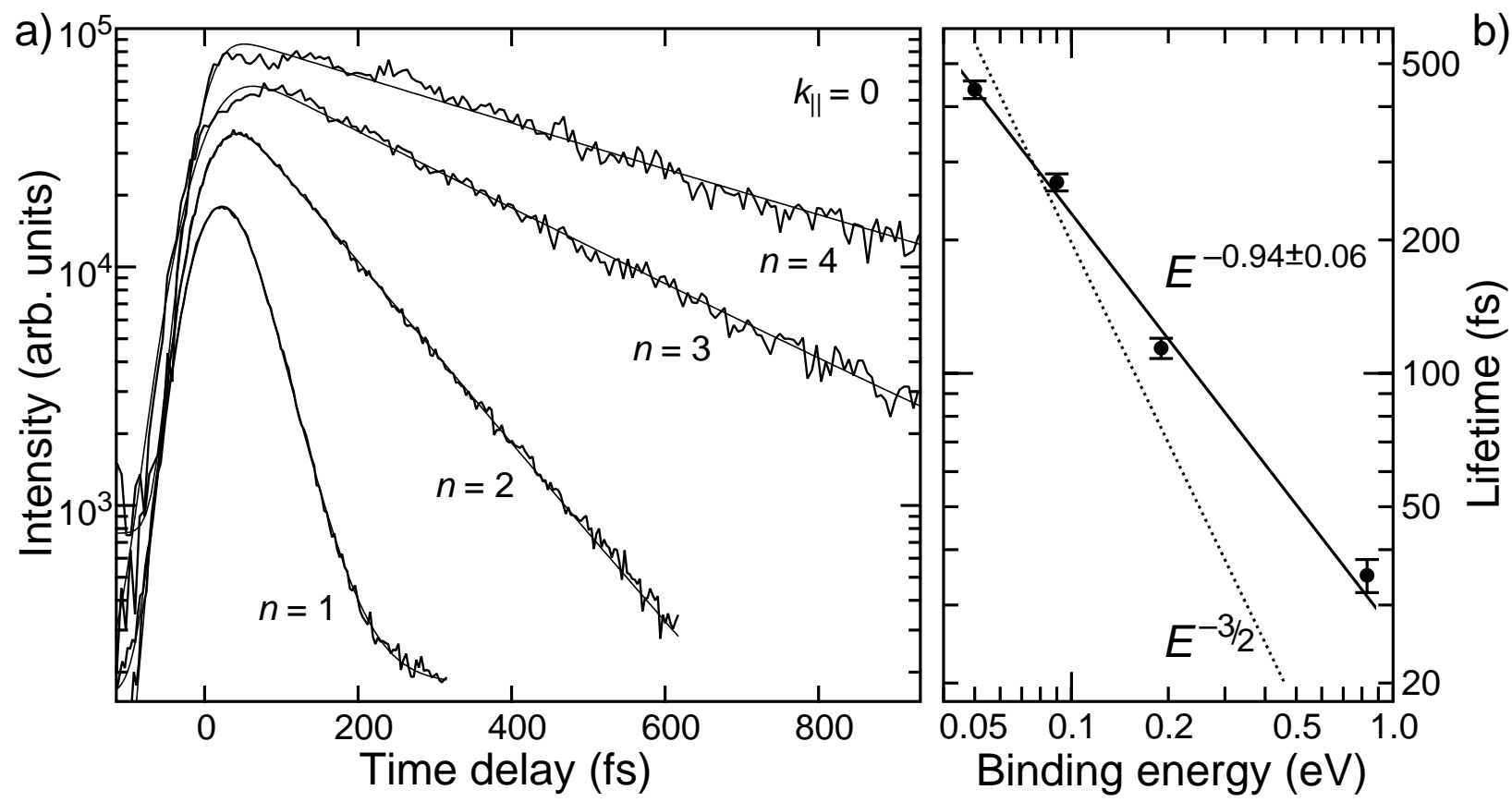

FIG. 3. a) Time-resolved measurements of the image-potential-state series at $k_{\|}=0$. b) Lifetimes as function of binding energy compared to various power-law dependencies (see text).

fact that the scattering model calculation neglects the round-trip phase shift $2 \phi_{g r}$ of the graphene layer. Using the expressions for the phase shift at the substrate and the imagepotential barrier [23] we obtain $\phi_{C}=0.63 \pi$ and $\phi_{B}=1.02 \pi$, respectively. The total phase shift for the $n=1$ state is $2 \pi$, from which we obtain $\phi_{g r}=0.18 \pi$. Note that such a small phase shift leads to a significant change in binding energy from $0.59 \mathrm{eV}$ to $0.83 \mathrm{eV}$ (see Table (I).

The time-resolved spectra of the image-potential states were also measured and are shown in Fig. 3a. As summarized in Table I, lifetimes of tens to hundreds of femtoseconds are obtained. These are comparable to values obtained for $\mathrm{Cu}(100)$ with a similar midgap image-potential state position and hence bulk evanescent decay length in the metal crystal [11]. Note, as an aside, that the curve measured at the energy of the $n=4$ image-potential state in Fig. [3a shows weak quantum beats [11] for delay times $<300$ fs. The data in Table [ show that lifetimes vary with binding energy approximately $\propto E^{-1}$ (solid line in Fig. 3b). The asymptotic, classical $\tau \propto E^{-3 / 2}$ behavior [24] (dashed line in Fig. 3b) is not reached for $n<4$. Similar behavior has been found on copper surfaces [25].

An important issue for carrier movement at graphene/metal interfaces is the degree of 


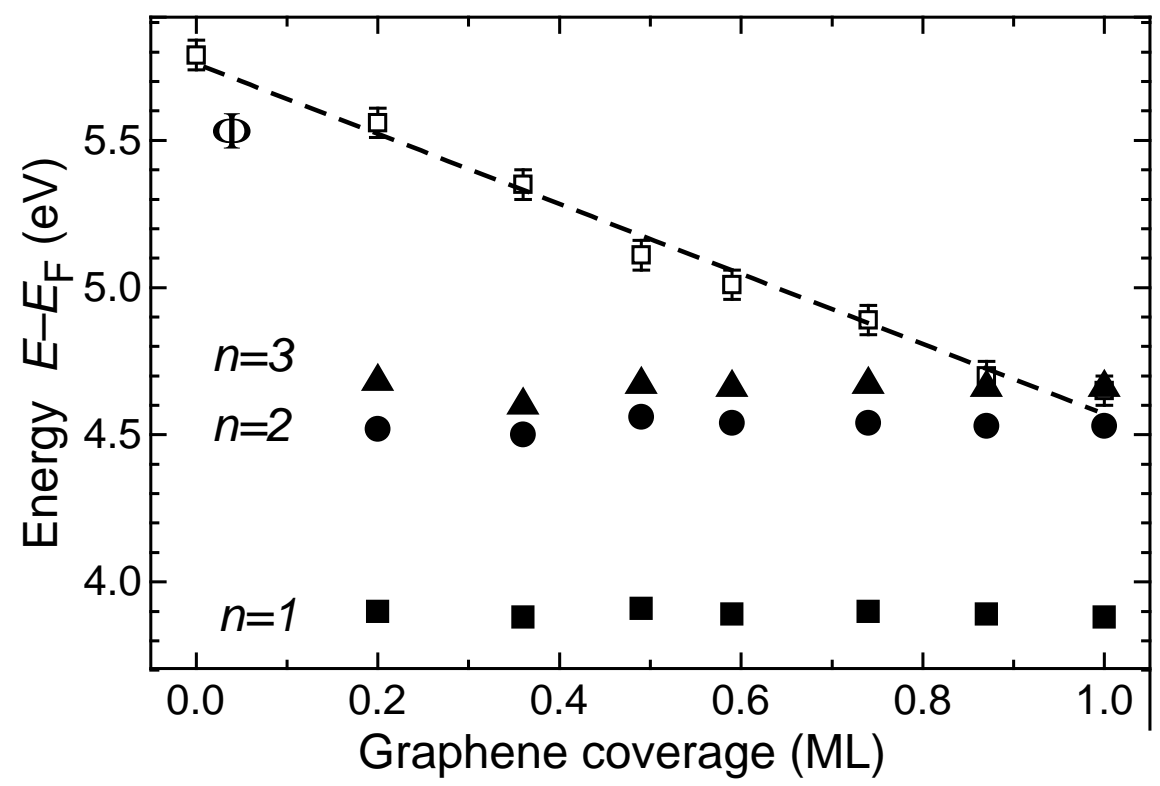

FIG. 4. Sample work function (open symbols) and image-potential states $n=1,2$, and 3 binding energies (filled symbols) as a function of graphene coverage. Dashed line represents a linear fit for the work-function change.

lateral confinement. This confinement can be examined at low graphene coverage, obtained via a small number of sequential TPG cycles. From previous studies, it is known that one TPG cycle covers a fraction of about $20 \%$ of uncovered Ir surface [17]. After one TPG cycle the typical island size is $(35 \mathrm{~nm})^{2}$ and after the second cycle of the order of $(100 \mathrm{~nm})^{2}$ [17].

As has been shown in earlier work [26], the average and local work function play an important role in interfacial electron localization. Thus the average work function $\Phi$ was measured via monochromatic $2 \mathrm{PPE}$ and the expression $\Phi=2 h \nu-\Delta E$, i.e., where $h \nu$ is the photon energy and $\Delta E$ is the difference between the Fermi level cutoff and the low-energy cutoff. Figure 4 displays the work function (open symbols) as a function of graphene coverage. The work function decreases approximately linearly from a value $5.79 \pm 0.10 \mathrm{eV}$ to $4.65 \pm 0.10 \mathrm{eV}$ from $\operatorname{Ir}(111)$ to $1 \mathrm{ML}$ graphene. Reported values of the work function for $\operatorname{Ir}(111)$ are 5.76 and $5.79 \mathrm{eV}$ [27]. The work function of the graphene-covered surface on $\operatorname{Ir}(111)$ is between the values for $\operatorname{Pt}(111)$ of $4.87 \mathrm{eV}$ and free-standing graphene of $4.48 \mathrm{eV}$ [28], which is consistent with the weak bonding between the $\operatorname{Ir}(111)$ and the graphene overlayer and a $p$-doping of the graphene [2]. The linear decrease of the work function is known for other systems and is due to the averaging over substrate and overlayer islands [26]. 
Image-potential states were observed at all coverages reported here using 2PPE. However, for the clean surface or uncovered substrate areas the available photon energies were not sufficient to populate image-potential states due to the large work function of $\operatorname{Ir}(111)$. The image-potential-state energies, measured relative to the Fermi level, are shown in Fig. 4. The energies are generally constant over the coverage range from 0.2 to $1 \mathrm{ML}$ with the intensity increasing monotonically with coverage. Note that the graphene Dirac cone at the $\mathrm{K}$ point has been clearly observed for more than three TPG cycles or $0.5 \mathrm{ML}$ graphene coverage [17]. The constant energy of the image-potential series as a function of coverage in Fig. 4 is a direct result of the localization of the electrons on the graphene islands [26]. The electrons respond to the local work function if the average island dimensions are larger than the typical distance of the probability density maximum which is of the order of nanometers for the lowest $n$ image-potential states. Note that the localization on the graphene islands is facilitated by the large work function difference between the graphene layers and the $\operatorname{Ir}(111)$ substrate. For small graphene islands, an energy shift proportional to $d^{-2}$, where $d$ denotes the characteristic island size, is expected due to the lateral localization of the electron in a two-dimensional quantum well [29]. However, these shifts would be $<1 \mathrm{meV}$ for the island sizes expected for the current preparation conditions [17].

In summary, we have observed and measured the properties of image-potential states on a graphene monolayer on $\operatorname{Ir}(111)$. The binding energy of the $n=1$ image-potential state is $40 \%$ larger than expected from the position of the graphene vacuum level relative to the $\operatorname{Ir}(111)$ band gap. There is no prominent indication of a second main series of image-potential states as predicted for free-standing graphene [1]. Apparently, the underlying metal substrate breaks the mirror symmetry of the graphene layer and the state of odd symmetry shifts up in energy as has been calculated for graphene on $\mathrm{Ru}(0001)$ [7]. In addition, the image-potential states can be excited efficiently from a downward dispersing Shockley surface state in the sp-band gap of the $\operatorname{Ir}(111)$ band structure indicating a sizable overlap of the wave functions of these states located at the substrate interface and graphene surface, respectively. The measured lifetimes of the image-potential states are comparable to similar clean metal surfaces. Recently, similar results have also been obtained for graphene on $\mathrm{Pt}(111)$ [30]. Apparently, the evanescent coupling of the image-potential-state wavefunctions to the underlying electronic states of the $\operatorname{Ir}(111)$ bulk and surface states is not altered by the graphene layer. Three-dimensional localization of electrons on graphene islands has 
been observed for submonolayer coverages obtained by individual TPG cycles. However, even for the smallest island size, no energy shift due to localization was observed within the experimental uncertainty. Further development is needed to prepare well-ordered graphene islands with controlled lateral extension. A different approach would be to exploit the moiré pattern on more corrugated graphene layers [7].

The work at Columbia University was supported under the US Department of Energy Contract No. DE-FG 02-04-ER-46157. The Zagreb group acknowledges support by the MZOS through project No. 035-0352828-2840 and MZOS-NSF support through contract No. 1/2009. M. K. thanks the Alexander von Humboldt Foundation for a research fellowship. We acknowledge helpful discussions with Branko Gumhalter.

[1] V. M. Silkin et al., Phys. Rev. B 80, 121408 (2009).

[2] I. Pletikosić et al., Phys. Rev. Lett. 102, 056808 (2009).

[3] Z. Sun et al., Phys. Rev. B 83, 081415 (2011).

[4] R. van Gastel et al., Appl. Phys. Lett. 95, 121901 (2009).

[5] F. Xia et al., Nature Nanotechnol. 6, 179 (2011).

[6] S. Bose et al., New J. Phys. 12, 023028 (2010).

[7] B. Borca et al., Phys. Rev. Lett. 105, 036804 (2010).

[8] Z. Hao et al., Phys. Rev. Lett. 105, 017602 (2010).

[9] K. Boger, Th. Fauster, and M. Weinelt, New J. Phys. 7, 110 (2005).

[10] H. Hattab et al., Appl. Phys. Lett. 98, 141903 (2011).

[11] U. Höfer et al., Science 277, 1480 (1997).

[12] P. M. Echenique and J. B. Pendry, J. Phys. C 11, 2065 (1978).

[13] J. Güdde et al., Science 318, 1287 (2007).

[14] C. R. Ast et al., Phys. Rev. Lett. 98, 186807 (2007).

[15] J. F. van der Veen, F. J. Himpsel, and D. E. Eastman, Phys. Rev. B 22, 4226 (1980).

[16] I. Pletikosić et al., J. Phys.: Condens. Matter 22, 135006 (2010).

[17] M. Kralj et al., Phys. Rev. B 84, 075427 (2011).

[18] D. Subramaniam et al., ArXiv e-prints (2011).

[19] See Supplemental Material at URL for data showing the resonant transition into th $n=2$ 
state.

[20] D. A. Papaconstantopoulos, Handbook of the Band Structure of Elemental Solids (Plenum Press, New York, 1986).

[21] Th. Fauster, Appl. Phys. A 59, 639 (1994).

[22] C. Busse et al., Phys. Rev. Lett. 107, 036101 (2011).

[23] N. V. Smith, Phys. Rev. B 32, 3549 (1985).

[24] Th. Fauster, Ch. Reuß, I. L. Shumay, and M. Weinelt, Chem. Phys. 251, 111 (2000).

[25] P. M. Echenique et al., Surf. Sci. Rep. 52, 219 (2004).

[26] R. Fischer et al., Phys. Rev. Lett. 70, 654 (1993), R. Fischer, Ph. D. thesis, Universität München, 1993.

[27] R. W. Strayer, W. Mackie, and L. W. Swanson, Surf. Sci. 34, 225 (1973), B. E. Nieuwenhuys, R. Bouwman, and W. M. H. Sachtler, Thin Solid Films 21, 51 (1974).

[28] G. Giovannetti et al., Phys. Rev. Lett. 101, 026803 (2008).

[29] R. Fischer, Th. Fauster, and W. Steinmann, Phys. Rev. B 48, 15496 (1993).

[30] D. Nobis, D. Niesner, and Th. Fauster (unpublished). 